\title{
Respiratory Motion Correction in Emission Tomography Image Reconstruction
}

\author{
Mauricio Reyes $^{1}$, Grégoire Malandain ${ }^{1}$, Pierre Malick Koulibaly ${ }^{2}$, \\ Miguel A. González Ballester ${ }^{3}$, and Jacques Darcourt ${ }^{2}$ \\ ${ }^{1}$ Epidaure team, INRIA Sophia Antipolis, France \\ \{Mauricio.Reyes_Aguirre,Gregoire.Malandain\}@sophia.inria.fr \\ ${ }^{2}$ Nuclear medicine department, Centre Antoine Lacassagne, Nice, France \\ ${ }^{3}$ MEM center, University of Bern, Switzerland
}

\begin{abstract}
In Emission Tomography imaging, respiratory motion causes artifacts in lungs and cardiac reconstructed images, which lead to misinterpretations and imprecise diagnosis. Solutions like respiratory gating, correlated dynamic PET techniques, list-mode data based techniques and others have been tested with improvements over the spatial activity distribution in lungs lesions, but with the disadvantages of requiring additional instrumentation or discarding part of the projection data used for reconstruction. The objective of this study is to incorporate respiratory motion correction directly into the image reconstruction process, without any additional acquisition protocol consideration. To this end, we propose an extension to the Maximum Likelihood Expectation Maximization (MLEM) algorithm that includes a respiratory motion model, which takes into account the displacements and volume deformations produced by the respiratory motion during the data acquisition process. We present results from synthetic simulations incorporating real respiratory motion as well as from phantom and patient data.
\end{abstract}

\section{Introduction}

Respiratory motion during the data acquisition process leads to blurred images, making difficult an accurate diagnosis, planning and following. For instance, mislocalizations of lesions in the fusion of positron emission tomography (PET) and computerized tomography (CT) have been found 1]. Similarly, significant tumor motion has been reported in others studies (e.g. [2]3]) as well as significant volume increase of lung lesions in images reconstructed without respiratory motion compensation [4].

To our knowledge, motion correction in Emission Tomography (ET) has been seldom investigated in the literature. Current methods can be classified in four main categories: post-processing, Multiple Acquisition Frame (MAF), sinogram data selection based on detected motion, and sinogram correction.

- Post-processing methods are based on transformations performed either in projection-space (e.g [5]) or in image-space (e.g. 6]). However, the motion models used in projection-space are too simplistic (e.g. global scaling), and 
the transformations applied in image-space do not consider the true effects of motion on the acquired data.

- The MAF-based methods consist on regrouping the projections in smaller subsets according to the detected motion (either online or offline motion detection). Then, image reconstruction of each subset is performed independently followed by a realignment of the images. These approaches present the inconvenient that the signal-to-noise ratio increases for images reconstructed from smaller subsets of projections.

- Sinogram data selection based on motion detection, also known as gating, has been used to compensate for motion correction in ET [4. Gating techniques have shown improvements, contributing to a better quantification of lesions. However, they require extra hardware or specific data acquisition modes and they discard part of the acquired projection data.

- Sinogram correction methodologies act directly on the projection data by repositioning the lines-of-response (LOR) when the motion is known 78 . However, these approaches are only applicable to rigid motions and require to deal with motion-corrected LOR's that may fall in non-valid positions, which decreases their practical interest.

The purpose of this paper is to describe a reconstruction algorithm that allows for a retrospective respiratory motion correction that operates directly over the complete projection data, without the need of additional acquisition protocols or discarding of data. To that end, we propose an extension to the MLEM reconstruction algorithm in which a motion model is plugged. We consider not only displacements but also local deformations.

The next sections present the methodology and results from phantom data and synthetic simulations incorporating real respiratory motion. For patient data, a preliminary approach of respiratory motion modelling and results of its use with the proposed motion correction methodology are also presented.

\section{Method}

\subsection{Maximum Likelihood Expectation Maximization}

First introduced in emission tomography by Shepp and Vardi 9], the MLEM algorithm is based on a Poisson model for the emission process. For a given emission element $b$ the number of emissions $f_{b}$ follows a Poisson law with mean $\lambda_{b}$. Besides, the projection matrix $R$ (or called by some authors system matrix or transition matrix) gives the probability that a certain emission from voxel $b$ is detected by the detector $d$ (called dexel hereafter). Furthermore, the number of detections from dexel $d$ (i.e., $p_{d}$ ) can be expressed in terms of the number of emissions $f_{b}, p_{d}=\sum_{b} f_{b} R_{d b}$. This latter expression is important since it states the relationship between detections and emissions through the system matrix values. Later, this fact will be exploited in the motion correction step.

We are interested to find the mean value $\lambda$ from the set of projections $p$. This can be done by searching the maximum likelihood of getting a set of measures $p$ 
given an image $\lambda$ (i.e., $\hat{\lambda}=\arg \max _{\lambda}[P(p \mid \lambda)]$ ). It can be shown (see [9] for more details) that $\hat{\lambda}$ can be found by means of an iterative algorithm

$$
\lambda_{b}^{<K+1>}=\frac{\lambda_{b}^{<K>}}{\sum_{d} R_{d b}} \sum_{d} \frac{p_{d} R_{d b}}{\sum_{b^{\prime}} \lambda_{b^{\prime}}^{<K>} R_{d b^{\prime}}}
$$

where $p_{d}$ stands for the number of detections of dexel $d, \lambda_{b}$ is the mean number of emissions from voxel $b, R_{d b}$ is the probability that a particle emitted from voxel $b$ is detected by the dexel $d$ and $K$ stands for the iteration number.

\subsection{Incorporating Motion Correction into the MLEM Algorithm}

We incorporate motion correction into the MLEM algorithm through the projection matrix $R$. We estimate the new contribution $R_{d b}^{C}$ of a voxel under motion $b$ to every dexel $d$.

Let us consider a continuous motion modeled by the spatial transformations $\varphi_{t}: \mathbb{R}^{3} \mapsto \mathbb{R}^{3}$, where $\varphi_{t}(m)$ denotes the position of point $m$ at time $t$. This motion is observed from time $t=0$ to $t=T$.

We first discretize this motion into a discrete set of spatial transformations $\varphi_{i}, i=0 \ldots N$, the transformation $\varphi_{i}$ being valid from $t=t_{i}$ to $t=t_{i+1} . R_{d b}^{C}$ expresses then the weighted sum of the contributions $R_{d b}^{i}$ of deformed voxels $\varphi_{i}(b)$ to $d$ :

$$
R_{d b}^{C}=\sum_{i} w_{i} R_{d b}^{i}
$$

The weights $w_{i}=\left(t_{i+1}-t_{i}\right) / T$ allow to take into account the kinetic of the motion: $w_{i} T$ represents the duration where $\varphi_{t}$ can be effectively approximated by $\varphi_{i}$.

\subsection{Computation of System Matrix Terms}

The voxels that contribute to a dexel $d$ are assumed to intersect a $3-\mathrm{D}$ line that stemed from $d$. Let us denote by $l_{d b}$ the length of the intersection of this line with the emission element $b$. We thus define the contribution of $b$ to $d$ by

$$
\text { static: } R_{d b}=\frac{l_{d b}}{\sum_{d^{\prime}} l_{d^{\prime} b}} \quad \text { dynamic: } R_{d b}^{i}=\frac{l_{d b}^{i}}{\sum_{d^{\prime}} l_{d^{\prime} b}^{i}} .
$$

In the static case, we model the emissions elements as spheres inscribed in the voxel space, which facilitates the calculation of Eq. (3): see Fig. 17. The summation in each denominator of Eq. (3) acts as a normalization term.

If no deformations can be assumed for emission elements $b$ during their motion, we could still have used the intersection of a line with a sphere for the computation of the contribution $R_{d b}^{i}$. However, this will not be realistic. Indeed, it has been shown that the displacements in the thorax (due to the respiratory motion) present a non-linear and a non-homogeneous behavior [3. Thus, we have to consider also the deformations of $b$. When under motion, the emission 


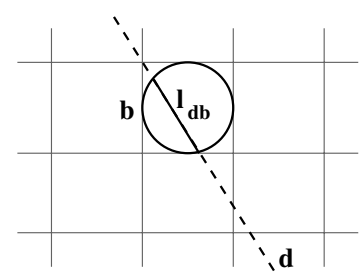

(a)

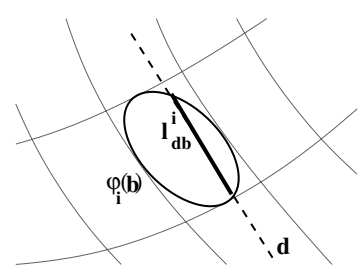

(b)

Fig. 1. The contribution of an emission element $b$ to a dexel $d$ represented by a dotted line is defined by the intersection (continuous line) of (a) a sphere with a line (static case) or (b) an ellipsoid (a deformed sphere) with a line (dynamic case)

element $b$ will deform into $\varphi_{i}(b), i=0 \ldots N$. As a first order approximation, a deformed sphere is an ellipsoid. The contribution of $b$ at state $i$ to $d$, i.e. $\varphi_{i}(b)$, is then similarly defined as the length intersection of the line $d$ with this ellipsoid (see Fig. 11).

The study of the matrix $\nabla \varphi_{i}$ allows to estimate the ellipsoid. Let us consider the singular value decomposition (SVD) of matrix $\nabla \varphi_{i}$, that is $\nabla \varphi_{i}=U \Sigma V^{T}$, where $U$ and $V$ are square and orthogonal matrices and $\Sigma=\operatorname{diag}\left(\lambda_{1}, \lambda_{2}, \lambda_{3}\right)$, with $\lambda_{j},(j=1,2,3)$ the singular values of $\nabla \varphi_{i}$. It turns out that the columns of $U$ are the eigenvectors of $\nabla \varphi_{i} \nabla \varphi_{i}{ }^{T}$, and also give the preferred local deformation directions, while the $\lambda_{j}$ are related to the magnitude of the deformations in the direction of the eigenvectors.

The modellization of the emissions elements as spheres that translate and deform locally into ellipsoids according to a known transformation, represents a novel contribution in this work. Furthermore, computations of the system matrix elements are faster than using classical methods of dexel-voxel intersection.

Since a dexel is defined by the path travelled by a single (e.g. SPECT) or by a pair of photons (e.g. PET), the method is independent of the type of ET modality, and thus can be used without further modifications.

\subsection{Estimation of the Respiratory Motion}

In practice, unless extra devices are used to measure the breathing pattern, the respiratory motion (transformation $\varphi$ ) is generally unknown.

A first approach to estimate this motion consisted in registering a known respiratory motion model on the data to be reconstructed. To build this model, two MRI images of a volunteer were acquired at breath holding in expiration and inspiration and then non-rigidly registered. This provides us with a volumic displacement vector field (DVF) $\mathbf{u}$. Transformations $\Phi_{i}(m)$ are then given by $\Phi_{i}(m)=m+i / N \mathbf{u}(m)$.

To adapt the transformation $\Phi_{i}$ to a patient, we first create an average image of the expiration and inspiration states (to simulate a non-corrected reconstruction) that is affinely registered against the non-corrected reconstructed patient's image. This provides us with an affine transformation $T$. We compose then the transformations to obtain $\varphi_{i}=T \circ \Phi_{i} \circ T^{-1}$. 
Though this method is by no means a robust method, it provides a first insight of the results that can be achieved by using such an approximative model with the proposed motion correction technique. Further improvements are needed to assure a good estimation of the patient's respiratory motion model.

\section{Results}

\subsection{Simulated Data}

We simulated respiratory motion in a SPECT study of lungs. For this, we used the thorax phantom NCAT (NURBS-based cardiac torso) [10], to which a small lesion of $15 \mathrm{~mm}$ diameter was added. The model was then deformed with $N \varphi_{i}$ transformations, estimated from a known real respiratory motion transformation $\Phi$. Sinograms were then computed for each time state using the SimSET (Simulation System for Emission Tomography) library and combined into one single sinogram by a weighted sum.

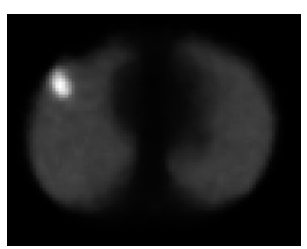

(a) Non-corrected

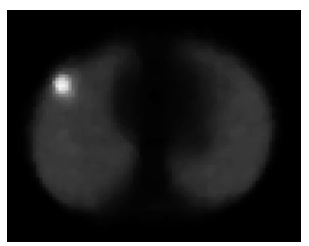

(b) Corrected

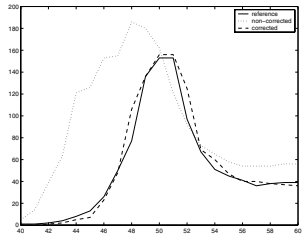

(c) Intensity profile

Fig. 2. Image reconstruction without motion correction (a) and with motion correction (b). The corrected profile (dashed line) show a close relationship with the reference profile (continuous line) in comparison with the non-corrected one (dotted line) (c).

Fig. 2 shows the reconstructed image with and without motion correction. As described in the literature, the lesion appears larger in the non-corrected reconstruction. A visual comparison of the intensity profiles (Fig. 2(c)) shows a good agreement between the motion-corrected reconstruction and the ground truth.

Two figures of merit were used to measure quantitatively the performance of the method, namely the coefficient of variability $C V=\sigma($ lesion $) / \mu($ lesion $)$ where $\mu$ (lesion) and $\sigma$ (lesion) denote the average and the standard deviation of the intensity values over the lesion, and the contrast recovery $C R=$ $\mu($ lesion $) / \mu$ (background) [1]. For the reference volume $\mathrm{CR}$ and $\mathrm{CV}$ values are 5.80 and 0.14 respectively. The non-corrected volume presents $\mathrm{CR}$ and $\mathrm{CV}$ values of 3.20 and 0.13 respectively. After motion correction the $\mathrm{CR}$ value increased to 4.40 while the $\mathrm{CV}$ value remained in 0.13 . From these results, it can be concluded that in the lesion area, noise properties are not affected by the motion correction. Higher $C R$ values are found for the corrected cases in comparison with the noncorrected one $(27 \pm 4 \%$ of increment), which demonstrates the deblurring effect of the motion correction. 


\subsection{Phantom Data}

A phantom made of three spheres filled with $99 \mathrm{mTc}$, having a concentration of $85 \mu \mathrm{Ci} / \mathrm{ml}$ each and of 1.8, 3.2 and $1.3 \mathrm{~cm}$ diameters (Inserts numbers 1, 2 and 3 respectively) was acquired with a Millenium-VG SPECT camera. Five data acquisitions were performed, and for each acquisition, the phantom was translated $1 \mathrm{~cm}$ in the axial direction. By combining the sinograms, we simulate the acquisition of a moving phantom. Finally, one single acquisition of duration five times longer was performed in the reference position, to serve as ground truth.

Reconstructed volumes had dimensions $128^{3}$ voxels with voxel size of 4.42 mm. Fig. 3 shows the corrected and non-corrected reconstructed volumes and the intensity profiles for insert number two.

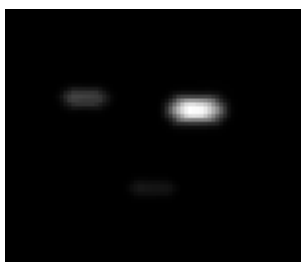

(a) Non-corrected

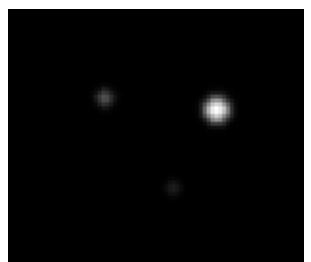

(b) Corrected

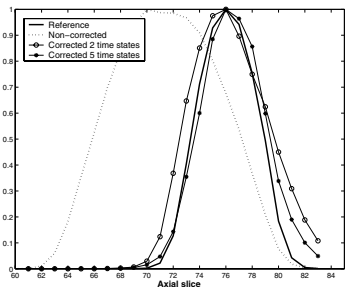

(c) Intensity profiles

Fig. 3. Axial translations of spherical sources during an ET study. Without motion correction (a), after motion correction (b) and the intensity profiles of reference, noncorrected and corrected volumes for insert number two (c).

For each insert, volume, $C R, C V$, Volume Error (VE), Volume Precision (VP), Centroid Error (CE) and Centroid Precision (CP) measurements were calculated to assess the quality of the motion correction in phantom data. VE and VP are defined as the relative error between the reference and non-corrected volumes and between the reference and corrected volumes, respectively. CE and $\mathrm{CP}$ are defined as the distance between reference and non-corrected centroids and between reference and corrected centroids, respectively. $C R$ ratios are presented as $C R_{c}$ (corrected contrast recovery) over $C R_{n c}$ (non-corrected contrast recovery). Similarly for $C V$, we have $C V_{c} / C V_{n c}$ (see Table1). From Table1 and Fig. 3 it can be seen that the motion correction method yields corrected volume size, spheres positions and improved contrast and noise properties.

Table 1. Results of motion correction for phantom data

\begin{tabular}{c|c|c|c|c|c|c}
\hline Insert & $\mathrm{VE}$ & $\mathrm{VP}$ & $\mathrm{CE}(\mathrm{cm})$ & $\mathrm{CP}(\mathrm{cm})$ & $C R_{c} / C R_{n c}$ & $C V_{c} / C V_{n c}$ \\
\hline 1 & $350 \%$ & $5,8 \%$ & 2.0 & 0.16 & 2.2 & 0.58 \\
\hline 2 & $125 \%$ & $1 \%$ & 1.96 & 0.21 & 1.62 & 0.89 \\
\hline 3 & $166 \%$ & $8 \%$ & 1.85 & 0.21 & 2.57 & 0.48 \\
\hline
\end{tabular}




\subsection{Patient Data}

Five patients, one lesion each, were used to test the methodology of motion correction in patient data. For each of them, image reconstruction with and without motion correction was performed. Gaussian regularization with filter full-width at half maximum (FWHM) of $8.5 \mathrm{~mm}$ every three iterations were set as main parameters. The reconstructed images had dimensions $128^{3}$ with voxel size of $4 \times 4 \times 4 \mathrm{~mm}^{3}$.

Table 2. Results of motion correction for patient dataset. The labels stand for: Noncorrected (NC), corrected (C), lateral (LR), anterior-posterior (AP) and cranial-caudal (CC).

\begin{tabular}{|c||c||ccc||cc||cc|}
\hline \multirow{2}{*}{ Patient } & \multicolumn{1}{|c||}{ Volume } \\
\cline { 3 - 9 } & $(\mathbf{C} / \mathbf{N C})$ & \multicolumn{3}{c||}{ Displacement $\mathbf{( m m )}$} & \multicolumn{2}{c||}{ CR } & \multicolumn{2}{c|}{ CV } \\
\cline { 3 - 9 } & LR & AP & CC & NC & C & NC & C \\
\hline 1 & 0.95 & 2.00 & 3.20 & 3.20 & 4.78 & 5.42 & 0.22 & 0.23 \\
\hline 2 & 0.64 & 2.60 & 3.60 & 5.10 & 5.04 & 6.06 & 0.24 & 0.20 \\
\hline 3 & 0.98 & 0.30 & 2.62 & 4.23 & 7.47 & 7.49 & 0.26 & 0.22 \\
\hline 4 & 0.86 & 0.45 & 1.20 & 1.74 & 3.66 & 3.90 & 0.18 & 0.16 \\
\hline 5 & 0.77 & 2.50 & 0.60 & 2.33 & 4.92 & 5.70 & 0.09 & 0.09 \\
\hline
\end{tabular}

From results presented in Table 2 it can be first noticed a volume reduction ranging from $2 \%$ to $36 \%$ after motion correction. Quantitative measures indicate improvements in contrast recovery after motion correction, which demonstrates the ability of the proposed method to compensate the blurring effects in the lesion area and its spatial activity distribution. Improvements in noise level are less significative. However, we did not find increases in noise level due to motion correction.

\section{Conclusion}

During an emission tomography study, induced motion due to patient breathing can lead to artifacts in the reconstructed image. This can produce less accurate diagnosis and more important, incorrect radiotherapy planning 34. We have presented a methodology to correct for respiratory motion in the image reconstruction step. The method accounts for a respiratory motion model that takes place in the computation of each term of the system matrix, and takes into account displacements and deformations experienced by the voxels during respiratory motion.

The method was implemented in a parallel framework and tested with simulated, phantom and patient data. For simulated and phantom data, the results show the ability of the proposed method to compensate for motion, rendering images with improved spatial intensity distributions and corrected lesions's shapes. For patient data, we have addressed the problem when no information about the patient's breathing cycle is available. As a first approach, we have used 
a respiratory motion model created by adapting a known model to the patient's anatomy. Although this method lacks of robustness, we are convinced that this first approach yields images with lesser respiratory motion effects than those reconstructed without motion correction. Improvements of the figures of merit were found after motion correction, and volume reduction and lesions displacements are likely to occur according to findings of previous studies [3].

Some further improvements and work in progress consider the inclusion of breathing and anatomy subject variability into the respiratory motion model estimation (i.e. transition from an individual respiratory motion model to a statistical one) and validation of the proposed motion correction method against a ground truth (e.g. gating).

\section{References}

1. MM. Osman, C. Cohade, Y. Nakamoto, LT. Marshall, JP. Leal, and RL. Wahl. Clinically significant inaccurate localization of lesions with PET/CT: frequency in 300 patients. J. Nucl. Med., 4(2):240-243, 2003.

2. S. Shimizu, H. Shirato, K. Kagei, T. Nishioka, X. Bo, H. Dosaka-Akita, S. Hashimoto, H. Aoyama, K. Tsuchiya, and K. Miyasaka. Impact of respiratory movement on the computed tomographic images of small lung tumors in threedimensional (3D) radiotherapy. Int J Radiat Oncol Biol Phys., 46(5):1127-33, Mar 2000 .

3. Y. Seppenwoolde, H. Shirato, K. Kitamura, S. Shimizu, M. van Herk M, JV. Lebesque, and K. Miyasaka. Precise and real-time measurement of 3D tumor motion in lung due to breathing and heartbeat, measured during radiotherapy. Int J Radiat Oncol Biol Phys., 53(4):822-34, July 2002.

4. SA. Nehmeh, YE. Erdi, KE. Rosenzweig, H. Schroder, SM. Larson, OD. Squire, and JL. Humm. Reduction of respiratory motion artifacts in PET imaging of lung cancer by respiratory correlated dynamic PET: Methodology and comparison with respiratory gated PET. J. Nucl. Med., 44(10):1644-1648, 2003.

5. W. Lu and TR. Mackie. Tomographic motion detection and correction directly in sinogram space. Phys. Med. Biol., 47(8):1267-84, April 2002.

6. O. Camara, G. Delso, and I. Bloch. Evaluation of a thoracic elastic registration method using anatomical constraints in oncology. In 2nd. Joint Conference of the IEEE Engineering in Medicine and Biology Society, 2002.

7. A. Rhamim and P. Bloomfield. Motion correction in histogram-mode and listmode EM reconstructions. In IEEE MIC, 2003.

8. K. Thielemans, S. Mustafovic, and L. Schnorr. Image reconstruction of motion corrected sinograms. In IEEE Medical Imaging Conf., 2003.

9. L. Shepp and Y. Vardi. Maximum likelihood reconstruction for emission tomography. IEEE Trans. Med. Imag., 1(2):113-122, 1982.

10. W.P. Segars. Development of a new dynamic NURBS-based cardiac-torso (NCAT) phantom. PhD thesis, The University of North Carolina, 2001.

11. R. Levkovitz, D. Falikman, M. Zibulevsky, A. Ben-Tal, and A. Nemirovski. The design and implementation of COSEM, an iterative algorithm for fully 3-D listmode data. IEEE Trans Med Imaging, 20(7):633-42, July 2001. 